\title{
EDTA-modified carbapenem inactivation method (eCIM) for detecting IMP Metallo- $\beta$ - lactamase-producing Pseudomonas aeruginosa: an assessment of increasing EDTA concentrations
}

\author{
Maxwell J. Lasko ${ }^{1}$, Christian M. Gill ${ }^{1}$, Tomefa E. Asempa ${ }^{1}$ and David P. Nicolau ${ }^{1,2^{*}}$ (D)
}

\begin{abstract}
Background: Prompt identification of carbapenemase-harboring organisms is valuable in informing therapeutic and infection-control measures. The modified carbapenem inactivation method (mCIM) and EDTA-modified carbapenem inactivation method (eCIM) are inexpensive and easy to interpret phenotypic tests endorsed by the Clinical and Laboratory Standards Institute (CLSI) for the detection of carbapenemase-harboring Enterobacterales. Only mCIM is endorsed by CLSI for detecting carbapenemase-harboring Pseudomonas aeruginosa. eCIM's ability to delineate serine and metallo- $\beta$-lactamases (MBL) could be advantageous in areas prevalent with carbapenemaseharboring $P$. aeruginosa. A recent assessment of $\mathrm{mCIM} / \mathrm{eCIM}$ on MBL-harboring $P$. aeruginosa demonstrated high eCIM sensitivity for NDMs and VIMs but not for IMP-producers. Therefore, this study aimed to determine whether increasing EDTA concentrations would enhance eCIM sensitivity for a collection of IMP-harboring P. aeruginosa isolates.

Twenty-six IMP-harboring $P$. aeruginosa isolates were utilized. For test validation, additional $P$. aeruginosa isolates harboring $\operatorname{NDM}(n=3), \operatorname{VIM}(n=3), \operatorname{KPC}(n=8)$, wild-type $(n=1)$, and Enterobacterales isolates harboring $\operatorname{IMP}(n=6)$ and NDM $(n=1)$ were assessed. The mCIM test was conducted as outlined by CLSI. Simultaneously, the eCIM test was performed with the standard $5 \mathrm{mM}$ EDTA concentration and doubling EDTA concentrations: $10 \mathrm{mM}, 20 \mathrm{mM}$, and $40 \mathrm{mM}$.
\end{abstract}

Results: Concentration-dependent improvement was observed among the IMP-harboring P. aeruginosa with eCIM sensitivities at 0,31, 85, and 100\% respectively. Remaining Enterobacterales and $P$. aeruginosa responded concordantly with their genotype at the standard $5 \mathrm{mM}$ eCIM concentration, with doubling EDTA concentrations providing no greater sensitivity.

Conclusion: Combination of mCIM and an eCIM with a 40 mM EDTA concentration appropriately capture IMPharboring $P$. aeruginosa without sacrificing test utility for other carbapenemase-harboring isolates.

Keywords: Carbapenemase, Resistance, Zinc-dependent, Metallo- $\beta$-lactamase, Pseudomonas aeruginosa

\footnotetext{
* Correspondence: david.nicolau@hhchealth.org

${ }^{1}$ Center for Anti-Infective Research and Development, Hartford Hospital, 80

Seymour Street, Hartford, CT 06102, USA

${ }^{2}$ Division of Infectious Diseases, Hartford Hospital, Hartford, CT, USA
}

\section{$\triangle B M C$}

(c) The Author(s). 2020 Open Access This article is licensed under a Creative Commons Attribution 4.0 International License, which permits use, sharing, adaptation, distribution and reproduction in any medium or format, as long as you give appropriate credit to the original author(s) and the source, provide a link to the Creative Commons licence, and indicate if changes were made. The images or other third party material in this article are included in the article's Creative Commons licence, unless indicated otherwise in a credit line to the material. If material is not included in the article's Creative Commons licence and your intended use is not permitted by statutory regulation or exceeds the permitted use, you will need to obtain permission directly from the copyright holder. To view a copy of this licence, visit http://creativecommons.org/licenses/by/4.0/ The Creative Commons Public Domain Dedication waiver (http://creativecommons.org/publicdomain/zero/1.0/) applies to the data made available in this article, unless otherwise stated in a credit line to the data. 


\section{Background}

Pseudomonas aeruginosa is a frequent cause of nosocomial infections and exhibits high intrinsic resistance to many common antimicrobials [1, 2]. Furthermore, horizontal transfer of genetic elements including carbapenemase genes can confer augmented resistance limiting treatment options [3]. Thus, the detection of carbapenemase-producing organisms is paramount for treatment decisions as well as infection control [4]. The Clinical and Laboratory Standards Institute (CLSI) endorsed modified carbapenem inactivation method (mCIM) and EDTA-modified carbapenem inactivation method (eCIM) have emerged as a combination phenotypic test to detect and differentiate between serine and metallo-based carbapenemases for Enterobacterales [5]. Their use of inexpensive products and easy to interpret results are favorable for clinical laboratories with limited resources.

A recent study assessing the utility of tandem mCIM/ eCIM against a variety of clinical metallo- $\beta$-lactamase (MBL)-producing $P$. aeruginosa isolates demonstrated high eCIM sensitivity for NDMs and VIMs but not for IMP-producers [6]. The poor sensitivity in differentiating
IMP-harboring $P$. aeruginosa isolates as metallo-based enzymes mirrors similar findings among IMP-harboring Enterobacterales [7]. The authors demonstrated $5 \mathrm{mM}$ provided optimal sensitivity compared to the initially investigated $0.1 \mathrm{mM}$ [7]. Therefore, this current study aimed to determine whether increasing EDTA concentrations would enhance eCIM sensitivity for a contemporary collection of IMP-harboring $P$. aeruginosa isolates.

\section{Results}

\section{$\mathrm{mCIM} / \mathrm{eCIM}$ evaluation}

Concordance between eCIM phenotypic tests and IMPharboring $P$. aeruginosa isolates was observed in $0 \%(0 /$ $26), 35 \%(9 / 26), 85 \%(22 / 26)$, and $100 \%(26 / 26)$ of isolates at EDTA concentrations of $5 \mathrm{mM}, 10 \mathrm{mM}, 20 \mathrm{mM}$, and $40 \mathrm{mM}$ respectively. Figure 1 illustrates a mCIM and eCIM test result for an IMP-harboring $P$. aeruginosa isolate. To complement percent concordance, eCIM zone sizes were recorded at each concentration for all utilized isolates, demonstrating an EDTA concentrationdependent effect for IMP-harboring $P$. aeruginosa isolates only. Notably, there were no major differences in

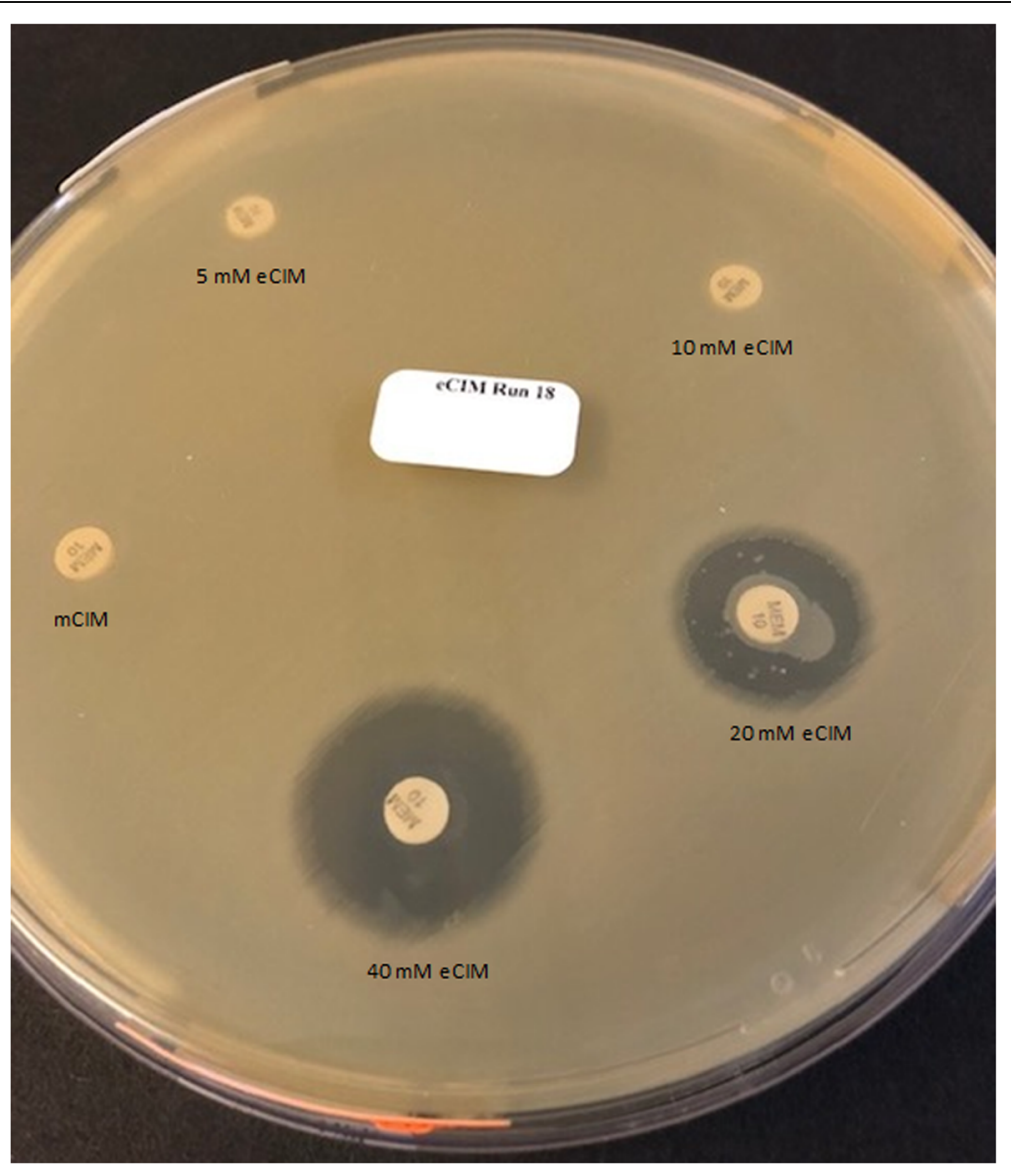

Fig. $1 \mathrm{mCIM} / \mathrm{eCIM}$ results for an IMP-48 harboring P. aeruginosa 
zone of inhibition diameter among different eCIM concentrations once an isolate's phenotypic result matched its genotype with an exception of one isolate. An IMP48 harboring isolate's zone of inhibition diameter increased from $12 \mathrm{~mm}$ to $26 \mathrm{~mm}$ as seen in Fig. 1. Moreover, 3 of the 4 P. aeruginosa isolates harboring IMP- 48 were false negative in the presence of $20 \mathrm{mM}$ EDTA, requiring the $40 \mathrm{mM}$ EDTA concentration to achieve $100 \%$ sensitivity (Table 1). NDM- and VIM-harboring $P$. aeruginosa and Enterobacterales isolates were eCIM true positive at the standard eCIM concentration of $5 \mathrm{mM}$, with doubling EDTA concentrations providing no greater sensitivity. As expected, all 8 serinecarbapenemase-harboring $P$. aeruginosa resulted in true negative eCIM results. Higher concentrations of EDTA had no detrimental effect on the growth or sensitivity of the test for any isolate.

\section{Establishment of controls}

The three evaluable control isolates were tested interday over 8 days. Observed eCIM results were 100\% concordant with their genotype. Thus, $P$. aeruginosa $27853, P$. aeruginosa \#0441, and $P$. aeruginosa \#0444 were utilized as controls throughout the study.

\section{Discussion}

At present, the standard eCIM test is a simple and inexpensive method for differentiating serine and metallo- $\beta$ lactamase activity in Enterobacterales. With an increased EDTA concentration of $40 \mathrm{mM}$, the eCIM test offers high sensitivity in differentiating between metallo- and serine- carbapenemase production among a diverse collection of $P$. aeruginosa. Improvements in eCIM sensitivity to IMP-producing $P$. aeruginosa are essential, given high prevalence rates, second only to VIM [8-10].

Similar improvements in eCIM sensitivity among Enterobacterales were observed in a study by Sfeir and colleagues [7]. An increase from $0.1 \mathrm{mM}$ to $5 \mathrm{mM}$ EDTA concentration increased eCIM sensitivity from 75 to $100 \%$. Of note, at $0.1 \mathrm{mM}$, all three IMP-positive Enterobacterales resulted as false negative, which was resolved at $5 \mathrm{mM}$. Another study evaluating the eCIM test with IMP-producing Enterobacterales demonstrated 55\% (6/
11) eCIM sensitivity with IMP-producing isolates, with the authors hypothesizing a higher EDTA concentration may be warranted [11]. Yamada and colleagues further evaluated several metal chelators, including EDTA, in conjunction with mCIM testing against IMP-positive Enterobacterales obtained from Japanese hospitals $(n=$ 93). An increase from $5 \mathrm{mM}$ to $10 \mathrm{mM}$ EDTA among IMP-positive Enterobacterales enhanced eCIM sensitivity from 79.6 to $98.9 \%$, again demonstrating an EDTA concentration-dependent improvement in sensitivity [12]. Notably, sub-genotypes were not reported in the aforementioned studies, and based on our findings with IMP-48, variability in the eCIM test performance may be enzyme-subtype specific and warrants additional investigation and reporting in future studies.

This study has limitations worth noting. No P. aeruginosa isolates evaluated in this study harbored both a metallo- $\beta$-lactamase and a serine carbapenemase. While rare, these dual carbapenemase-harboring isolates would likely result in a false negative eCIM interpretation regardless of EDTA concentration and remains an inherent limitation of the eCIM test. A multi-center validation study using an increased EDTA methodology is warranted.

\section{Conclusion}

In summary, we observed an EDTA concentrationdependent improvement in eCIM sensitivity among IMP-harboring $P$. aeruginosa, providing an important contribution to optimization of this phenotypic test. At a $40 \mathrm{mM}$ EDTA concentration, the eCIM provides high sensitivity for differentiating between metallo-dependent and serine carbapenemase-producing $P$. aeruginosa and provides clinical laboratories a reliable and accurate phenotypic screening assay.

\section{Methods \\ Bacterial isolates}

Forty-eight clinical isolates were utilized including 26 IMP-harboring $P$. aeruginosa isolates. For test validation, additional $P$. aeruginosa isolates harboring $\mathrm{NDM}(n=3)$, VIM $(\mathrm{n}=3), \operatorname{KPC}(n=8)$, wild-type $(n=1)$ as well as Enterobacterales isolates harboring $\operatorname{IMP}(n=6)$ and

Table 1 Sensitivity and Specificity of eCIM at Respective EDTA Concentrations

\begin{tabular}{llll}
\hline & IMP-harboring P. aeruginosa Only & All Evaluable Isolates & \\
\cline { 3 - 4 } eCIM Concentration & Sensitivity $[n=26](95 \% \mathrm{Cl})$ & $33 \%(19-50 \%)$ & Specificity $[n=8](95 \% \mathrm{Cl})$ \\
\hline 5 mM EDTA & $0 \%(0-15 \%)$ & $56 \%(40-72 \%)$ & $100 \%(63-100 \%)$ \\
$10 \mathrm{mM}$ EDTA & $35 \%(17-56 \%)$ & $90 \%(76-97 \%)$ & $100 \%(63-100 \%)$ \\
$20 \mathrm{mM}$ EDTA & $85 \%(65-96 \%)$ & $100 \%(91-100 \%)$ & $100 \%(63-100 \%)$ \\
$40 \mathrm{mM}$ EDTA & $100 \%(87-100 \%)$ & $100 \%(63-100 \%)$
\end{tabular}

a All evaluable isolates consist of $P$. aeruginosa harboring IMP, NDM, VIM, and KPC, as well as Enterobacterales harboring IMP and NDM. WT was not included in eCIM sensitivity or specificity analysis 
NDM $(\mathrm{n}=1)$ were assessed. Fourteen were acquired from the Centers for Disease Control and Food and Drug Administration Resistance Bank (CDC and FDA$\mathrm{ARB})$ and the remaining from the Center of AntiInfective Research and Development isolate library. Isolates were previously categorized by PCR or whole genome sequencing for the detection of $\beta$-lactamase producing genes. Evaluated enzyme subtypes harbored among evaluated $P$. aeruginosa isolates included IMP ($1,-6,-7,-10,-18,-48,-62)$, VIM $(-2,-5)$, NDM $(-1)$, and $\mathrm{KPC}(-2,-5)$. Evaluated Enterobacterales isolates harbored IMP $(-1,-4,-8)$ and $\operatorname{NDM}(-1)$. Meropenem MICs for carbapenemase-harboring $P$. aeruginosa were $>8 \mu \mathrm{g} / \mathrm{mL}$.

All isolates were stored in skim milk (Becton Dickinson, Sparks, MD) at $-80{ }^{\circ} \mathrm{C}$ and subcultured to Trypticase soy agar with 5\% sheep blood plates (Becton Dickinson, Sparks, MD) prior to incubation. Isolates were incubated, without selective disc pressure, at $37^{\circ} \mathrm{C}$ for $18-20 \mathrm{~h}$ prior to second subculture before testing.

\section{Evaluation of $\mathrm{mCIM} / \mathrm{eCIM}$}

The mCIM test was conducted as previously described for $P$. aeruginosa $[5,13,14]$. Briefly, $2 \mathrm{~mL}$ of trypticase soy broth were inoculated with a $10-\mu \mathrm{L}$ loopful of $P$. aeruginosa, vortexed, and a $10 \mu \mathrm{g}$ meropenem disk (Becton Dickinson, Sparks, MD, LOT: 9065664 and 9186033) was placed into the mixture. This mixture incubated for $4 \mathrm{~h}( \pm 15 \mathrm{~min})$. Following incubation, the meropenem disk was removed from the tube and placed on a Mueller-Hinton agar plate that was lawned with a 0.5 McFarland suspension of Escherichia coli ATCC 25922. Simultaneously, the eCIM test was performed with the standard $5 \mathrm{mM}$ EDTA concentration, as well as doubling EDTA concentrations (i.e. $10 \mathrm{mM}, 20 \mathrm{mM}$, and $40 \mathrm{mM}$ ). Isolates were run in duplicate at each concentration and zone diameters of inhibition were measured by two independent investigators. eCIM was interpreted as positive (detection of metallo- $\beta$-lactamase) if the zone of inhibition diameter increases by $\geq 5 \mathrm{~mm}$ compared with the isolate's mCIM reading and was considered negative if the zone of inhibition diameter was $\leq 4 \mathrm{~mm}$.

Data analysis was conducted in SPSS (IBM. Armonk, $\mathrm{NY}$ ). Isolates' phenotypic tests were compared to the genotypic standard. An isolate was defined as eCIM true positive if the phenotypic test matched an MBL-positive genotype or eCIM true negative if the phenotype matched a serine carbapenemase-positive genotype. eCIM false-positive results were defined as a phenotype indicating MBL production (eCIM positive) in isolates with negative genotypic findings and an eCIM falsenegative result was defined as a negative phenotypic result (eCIM negative) despite presence of an MBL gene.

\section{Establishment of controls}

Wild type $P$. aeruginosa isolate (ATCC 27853) served as a carbapenemase mCIM control. In order to establish appropriate and publically-accessible eCIM controls for use with $P$. aeruginosa, one KPC-harboring isolate (\#0441) and one VIM-harboring P.aeruginosa isolate (\#0444) from the CDC and FDA-AR isolate bank were utilized. Quality control testing was performed on each testing day.

\section{Abbreviations \\ mCIM: Modified carbapenem inactivation method; eCIM: EDTA-modified carbapenem inactivation method; CLSI: Clinical and Laboratory Standards Institute; MBL: Metallo- $\beta$-lactamase; NDM: New Delhi metallo- $\beta$-lactamase; VIM: Verona integron-encoded metallo- $\beta$-lactamase; IMP: Imipenemase; KPC: Klebsiella pneumoniae carbapenemase; WT: Wild type; CDC: Centers for Disease Control; FDA-AR: Federal Drug and Administration Antibiotic Resistance Bank}

\section{Acknowledgments}

We thank The Center for Anti-Infective Research and Development staff for their assistance.

\section{Authors' contributions}

Conceived and designed experiments: DPN. Performed experiments: MJL and CMG. Analyzed and Interpreted the Data: MJL, CMG, TEA, DPN. Wrote the manuscript: MIL, CMG, TEA, DPN. All authors read and approved the final manuscript.

\section{Funding}

This project was internally funded. External funding bodies had no role in the study design, data collection, analysis, interpretation or writing of the manuscript.

\section{Availability of data and materials}

The datasets used and/or analysed during the current study are available from the corresponding author on reasonable request.

Ethics approval and consent to participate

Not applicable.

\section{Consent for publication}

Not applicable.

\section{Competing interests}

DPN served as a consultant, speaker's bureau member or have received research funding from: Allergan, Bayer, Cepheid, Merck, Melinta, Pfizer, Wockhardt, Shionogi, Tetraphase. MJL, CMG, TEA have no conflicts to disclose.

Received: 13 April 2020 Accepted: 14 July 2020

Published online: 20 July 2020

References

1. Lister PD, Wolter DJ, Hanson ND. Antibacterial-resistant Pseudomonas aeruginosa: clinical impact and complex regulation of chromosomally encoded resistance mechanisms. Clin Microbiol Rev. 2009;22(4):582-610.

2. Rodríguez-Martínez J-M, Poirel L, Nordmann P. Molecular epidemiology and mechanisms of Carbapenem resistance in Pseudomonas aeruginosa. Antimicrob Agents Chemother. 2009;53(11):4783-8.

3. Oliver A, Mulet X, López-Causapé C, Juan C. The increasing threat of Pseudomonas aeruginosa high-risk clones. Drug Resist Updat. 2015;21-22: 41-59.

4. Guidelines for the Prevention and Control of Carbapenem-Resistant Enterobacteriaceae, Acinetobacter baumannii and Pseudomonas aeruginosa in Health Care Facilities. Geneva: World Health Organization; 2017. Available from: https://www.ncbi.nlm.nih.gov/books/NBK493061/. Accessed December 2019. 
5. CLSI. Performance standards for antimicrobial susceptibility testing. 29th ed. Wayne, PA: Clinical and Laboratory Standards Institute; 2019.

6. Gill CM, Lasko MJ, Asempa TE, Nicolau DP. Evaluation of the EDTA-Modified Carbapenem Inactivation Method (eCIM) for Detecting Metallo- $\beta$-lactamaseproducing Pseudomonas aeruginosa. J Clin Microbiol. 2020;58(6):e02015-19.

7. Sfeir MM, Hayden JA, Fauntleroy KA, Mazur C, Johnson JK, Simner PJ, et al. EDTA-modified Carbapenem inactivation method: a phenotypic method for detecting Metallo- $\beta$-lactamase-producing Enterobacteriaceae. J Clin Microbiol. 2019;57(5):e01757-18.

8. Hong DJ, Bae IK, Jang IH, Jeong SH, Kang HK, Lee K. Epidemiology and characteristics of Metallo- $\beta$-lactamase-producing Pseudomonas aeruginosa. Infect Chemother. 2015;47(2):81-97.

9. Kazmierczak KM, Rabine S, Hackel M, McLaughlin RE, Biedenbach DJ, Bouchillon SK, et al. Multiyear, multinational survey of the incidence and global distribution of Metallo- $\beta$-lactamase-producing Enterobacteriaceae and Pseudomonas aeruginosa. Antimicrob Agents Chemother. 2016;60(2):106778.

10. Kizny Gordon AE, Mathers AJ, Cheong EYL, Gottlieb T, Kotay S, Walker AS, et al. The hospital water environment as a reservoir for Carbapenemresistant organisms causing hospital-acquired infections-a systematic review of the literature. Clin Infect Dis. 2017;64(10):1435-44.

11. Liao Q, Xie Y, Wang C, Zong Z, Wu S, Liu Y, et al. Development and evaluation of the method for detecting metallo-carbapenemases among carbapenemase-producing Enterobacteriaceae. J Microbiol Methods. 2019; 163:105652.

12. Yamada K, Sasaki M, Imai W, Murakami H, Morita T, Aoki K, et al. Evaluation of inhibitor-combination $\mathrm{mCIM}$ for detecting MBL-producing Enterobacterales using three MBL inhibitors. J Med Microbiol. 2019;68(11): $1604-6$.

13. Lisboa LF, Turnbull L, Boyd DA, Mulvey MR, Dingle TC. Evaluation of a modified Carbapenem inactivation method for detection of Carbapenemases in Pseudomonas aeruginosa. J Clin Microbiol. 2018;56(1): e01234-17.

14. Simner PJ, Opene BNA, Chambers KK, Naumann ME, Carroll KC, Tamma PD. Carbapenemase detection among Carbapenem-resistant glucosenonfermenting gram-negative bacilli. J Clin Microbiol. 2017;55(9):2858-64.

\section{Publisher's Note}

Springer Nature remains neutral with regard to jurisdictional claims in published maps and institutional affiliations.

Ready to submit your research? Choose BMC and benefit from:

- fast, convenient online submission

- thorough peer review by experienced researchers in your field

- rapid publication on acceptance

- support for research data, including large and complex data types

- gold Open Access which fosters wider collaboration and increased citations

- maximum visibility for your research: over $100 \mathrm{M}$ website views per year

At $\mathrm{BMC}$, research is always in progress.

Learn more biomedcentral.com/submissions 